\title{
Genetic determination of fatty acid composition in Spanish Churra sheep milk
}

\author{
J. P. Sánchez, ${ }^{* 1}$ F. San Primitivo, ${ }^{*}$ E. Barbosa, ${ }^{*} \dagger$ L. Varona, $\neq$ and L. F. de la Fuente ${ }^{*}$ \\ *Departamento de Producción Animal, Universidad de León, León 24071, Spain \\ †Instituto Politécnico de Viana do Castelo, Escola Superior Agrária de Ponte de Lima, Portugal \\ fUnidad de Genética Cuantitativa y Mejora Animal, Universidad de Zaragoza, Zaragoza 50013, Spain
}

\begin{abstract}
The objective of this study was to estimate the genetic variation of ovine milk fatty acid (FA) composition. We collected 4,100 milk samples in 14 herds from 976 Churra ewes sired mostly by 15 AI rams and analyzed them by gas-liquid chromatography for milk fatty acid composition. The studied traits were 12 individual FA contents (proportion in relation to the total amount of FA), 3 groups of fatty acids [saturated fatty acids (SFA), monounsaturated FA (MUFA), and polyunsaturated FA (PUFA)], and 2 FA ratios (n-6:n-3 and C18:2 cis-9,trans-11:C18:1 trans-11). In addition, percentages of fat and protein and daily milk yield were studied. For the analysis, repeatability animal models were implemented using Bayesian methods. In an initial step, univariate methods were conducted to test the hypothesis of the traits showing additive genetic determination. Deviance information criterion and Bayes factor were employed as model choice criteria. All the studied SFA showed additive genetic variance, but the estimated heritabilities were low. Among unsaturated FA (UFA), only C18:1 trans-11 and C18:2 cis-9,cis-12 showed additive genetic variation, their estimated heritabilities being [marginal posterior mean (marginal posterior SD)] $0.02(0.01)$ and $0.11(0.04)$, respectively. For the FA groups, only PUFA showed significant additive genetic variation. None of the studied ratios of FA showed additive genetic variation. In second multitrait analyses, genetic correlations between individual FA and production traits, and between groups of FA and ratios of FA and production traits, were investigated. Positive genetic correlations were estimated among medium-chain SFA, ranging from 0 to 0.85 , but this parameter was close to zero between long-chain SFA (C16:0 and C18:0). Between long- and medium-chain SFA, estimated genetic correlations were negative, around -0.6. Among those UFA showing significant additive genetic variance, genetic correlations were close to zero. The estimated genetic correlations among
\end{abstract}

Received April 1, 2009.

Accepted September 15, 2009.

${ }^{1}$ Corresponding author: jpsans@unileon.es all the investigated FA, milk yield, and fat and protein percentages were not different from zero. Our results suggest that low additive genetic variation is involved in the determination of the FA composition of milk fat in Churra sheep under current production conditions, which results in low values of heritabilities.

Key words: dairy sheep, fatty acid, genetic parameter, milk composition

\section{INTRODUCTION}

Today, the human diet is regarded as ideal if it includes 30\% saturated fatty acids (SFA), $60 \%$ monounsaturated fatty acids (MUFA), and 10\% polyunsaturated fatty acids (PUFA; Pascal, 1996). Diets with this fatty acid (FA) composition have positive effects on human health. The FA profile in sheep milk is far from having this composition: SFA are higher in proportion (66\%), and MUFA and PUFA are lower (28 and $6 \%$, respectively; Cabiddu et al., 2005). Research has been carried out to discover management and breeding strategies for effectively modifying the FA profile of ruminant products. In addition, because some PUFA have shown highly desirable biological activity (i.e., conjugated linoleic acid), they have been given more attention in studies.

\section{Conjugated Linoleic Acid}

Conjugated linoleic acid (CLA; C18:2 cis-9,trans-11) is abundant in ruminant products. It has been proven to prevent cancer and chronic diseases (Khanal and Dhiman, 2004), as demonstrated in several animal models (Mitchell and McLeod, 2008) and also in humans, the results in the latter case being less conclusive (Tricon et al., 2004; Tricon and Yaqoob, 2006). The origin of this FA in milk is always from biosynthesis, either by rumen bacteria or by endogenous synthesis in the mammary gland or adipose tissues. Apparently, most CLA in milk is of endogenous origin. But both sources of CLA biosynthesis are related to each other because a high proportion of the trans-vaccenic acid used during endogenous biosynthesis had been generated as a subproduct of rumen bacteria biohydrogenation of linoleic 
or linolenic acids. For a review of metabolic pathways in the biosynthesis of CLA, see Khanal and Dhiman (2004).

Different sources of genetic variation could be involved in CLA biosynthesis a priori; the most important is thought to occur at $\Delta^{9}$-desaturase enzyme level. Several polymorphisms have been reported in the gene coding this enzyme within and across dairy cattle breeds (Milanesi et al., 2008); variation in the expression of the gene across breeds has also been reported (Taniguchi et al., 2004). This evidence supports the hypothesis that $\Delta^{9}$-desaturase activity may show additive genetic variation useful in breeding programs. Genetic variation could also exist at the level of the amount and activity of that bacteria involved in rumen biosynthesis (i.e., Butyrivibrio fibrisolvens and related bacteria) and, with regard to this, it has been shown that individual variation exists in the composition of bacteria flora in sheep (Swain et al., 1996).

\section{FA Profile in Milk}

Several factors affect the overall FA profile in milk, and it seems that diet is the most important one. De La Fuente et al. (2009) found indirectly that factors closely associated with feed (e.g., the combined effect of herd and season of the year) explained the majority of phenotypic variation of FA contents. In this sense, it has been shown that throughout feeding, FA composition of milk can be effectively altered to obtain a FA profile closer to that regarded as ideal: an increased proportion of MUFA and PUFA and a reduced percentage of SFA (Cabiddu et al., 2005; Gómez-Cortés et al., 2008).

Some FA in milk fat (in particular, PUFA and MUFA, which are of greater interest from a dietetic point of view) are generated by biosynthesis and, because different enzymes are involved in these processes, the same kind of arguments as for CLA could be posed for hypothesizing the existence of additive genetic variation. It has been demonstrated in dairy cattle that FA profile shows individual variation and that this variation partly has an additive genetic origin (Mele et al., 2007, 2009; Soyeurt et al., 2007; Bobe et al., 2008; Stoop et al., 2008), indicating that selective breeding could be a useful tool for improving the dietetic qualities of milk fat.

For dairy sheep, no studies have been carried out on quantifying the amount of additive genetic variance involved in the determination of the FA profile of milk. The objective of this study was to determine genetic variance and covariance for ovine milk FA composition for the first time, using dairy Churra sheep, to evaluate the possibility of using selective breeding as a tool for modifying FA profile in milk fat of this breed.

\section{MATERIALS AND METHODS}

\section{Data}

A total of 4,100 records from 976 Churra ewes (De La Fuente et al., 1995) were collected in 14 herds that were widely genetically connected through AI. An average of 69.7 ewes in each flock (ranging from 25 to 187) had records. The average number of records per female was 4.20 , reaching a maximum of 12 . Out of the total number of females with data, 906 were sired by 15 AI rams with an average family size of 65.1 daughters, ranging from 32 to 157 . Paternities were not known for the remaining 70 females. In addition to this paternity information, 2 more generations of ancestors were included from the historical pedigree file provided by ANCHE (Spanish Churra Breeders Association), so the final pedigree file consisted of 3,524 records.

Sample collection took place between January 2006 and June 2007 in herds distributed throughout Castile and León (Spain). Samples were obtained from a single morning or evening milking session, and a particular animal could be sampled several times per lactation for up to 2 consecutive lactations. Of the samples, $14 \%$ were obtained for ewes younger than $1 \mathrm{yr}$ of age, $31 \%$ from 1-yr-old females, $25 \%$ from 2-yr-old females, $16 \%$ from 3 - to 4-yr-old females, and $9 \%$ from females older than $4 \mathrm{yr}$ of age; the remaining $4 \%$ of the samples were taken from females whose age was not known. Regarding the distribution of records within the lactation period, $7 \%$ of the samples were obtained during the first month of lactation, $22 \%$ during the second, $26 \%$ during the third, $23 \%$ during the fourth, $11 \%$ during the fifth, and $10 \%$ during the sixth. The average size of contemporary group (number of females sampled in the same herd and day combination) was 26.1, ranging from 6 to 83 .

Milk fat extraction was carried out using the method by Bligh and Dyer (1959). The FA content was then determined by gas chromatography after methylation of the free FA with $\mathrm{NaOCH}_{3}$, and a Hewlett Packard 6890 Series GC System chromatographer (Palo Alto, CA) was used to measure FA contents. More details on the analytical procedure for measuring FA contents can be found in De La Fuente et al. (2009). To validate the analytical procedure, a few samples were processed several times and the analytical repeatabilities for individual fatty acids were computed. They were between 0.6 and 0.98 , and CLA had a repeatability of 0.95 .

\section{Traits}

The contents of $12 \mathrm{FA}$, as a percentage in relation to the total content of analyzed FA, were studied (Table 
Table 1. Descriptive statistics for the studied traits

\begin{tabular}{lrrrr}
\hline Trait $^{1}$ & $\mathrm{n}$ & Mean & $\mathrm{SD}$ & $\mathrm{CV}$ \\
\hline C8:0 0 & 4,100 & 3.45 & 0.46 & 13.29 \\
C10:0 & 4,100 & 8.60 & 1.40 & 16.26 \\
C12:0 & 4,100 & 5.37 & 1.13 & 21.12 \\
C14:0 & 4,100 & 10.18 & 1.45 & 14.28 \\
C16:0 & 4,100 & 22.05 & 2.42 & 11.00 \\
C18:0 & 4,100 & 10.48 & 2.20 & 20.95 \\
C16:1 cis-9 & 4,100 & 1.64 & 0.34 & 20.78 \\
C18:1 trans-11 & 4,100 & 2.53 & 0.46 & 18.14 \\
C18:1 cis-9 & 4,100 & 15.36 & 2.25 & 14.66 \\
C18:2 cis-9, cis-12 12.100 & 3.47 & 0.62 & 17.85 \\
C18:3 cis-9, cis-12, cis-15 & 4,100 & 1.23 & 0.54 & 43.68 \\
C18:2 cis-9, trans-11 (CLA) & 4,100 & 0.89 & 0.44 & 48.87 \\
SFA & 4,100 & 71.33 & 3.53 & 4.95 \\
MUFA & 4,100 & 22.11 & 2.88 & 13.00 \\
PUFA & 4,100 & 6.55 & 1.28 & 19.55 \\
n-6:n-3 3 CLA:1 trans-11 & 4,100 & 3.98 & 2.11 & 53.06 \\
Protein (\%) & 4,100 & 0.35 & 0.15 & 42.26 \\
Fat $(\%)$ & 3,448 & 5.47 & 0.75 & 13.76 \\
Milk yield (mL/d) & 3,448 & 6.57 & 1.86 & 28.34 \\
\hline
\end{tabular}

${ }^{1} \mathrm{CLA}=$ conjugated linoleic acid; SFA = saturated fatty acids (FA); MUFA = monounsaturated FA; PUFA = polyunsaturated FA. The content of FA is expressed as a percentage in relation to the total amount of FA. n- 6 includes C18:2 cis-9, cis-12, C18:3 cis-6, cis-9, cis-12, and C20:4 cis-5, cis-8, cis-11, cis-14; n-3 includes C18:3 cis-9, cis-12, cis-15.

1). Also, 3 groups of fatty acids were created and studied: SFA, which included even-chain FA between C4:0 and $\mathrm{C} 24: 0$ as well as odd-chain FA C13:0 to C17:0 and C21:0 to C23:0; MUFA, which included cis isomers of $\mathrm{C} 14: 1$ cis-9 and $\mathrm{C} 16: 1$ cis-9 to $\mathrm{C} 20: 1$ cis-9 as well as both cis and trans isomers of vaccenic acid (C18:1 trans-11 and C18:1 cis-11) and C18:1 cis-12; and PUFA, which included cis and trans isomers of linoleic acid (C18:2 cis-9,cis-12 and C18:2 trans-9,trans-12), 2 position isomers of CLA (C18:2 cis-9,trans-11 and C18:2 trans-10,cis-12), $\alpha$ - and $\gamma$-linolenic acids (C18:3 cis9,cis-12,cis-15 and C18:3 cis-6,cis-9,cis-12), and C20:4 cis-5,cis-8,cis-11,cis-14. (Note that in these groups some of the included FA were not studied individually.) Finally, 2 ratios between FA were considered: 1) n-6:n-3, where n-6 included C18:2 cis-9,cis-12, C18:3 cis-6, cis9,cis-12, and C20:4 cis-5,cis-8,cis-11,cis-14 and n-3 was C18:3 cis-9,cis-12,cis-15; and 2) C18:2 cis-9,trans-11 (CLA):C18:1 trans-11. This ratio was considered as a proxy of $\Delta^{9}$-desaturase activity in relation to the endogenous biosynthesis of CLA. Protein and fat percentages and daily milk yield were also studied. Table 1 shows descriptive statistics for all of these traits.

\section{Methods}

Univariate Models for Testing the Hypothesis of Additive Genetic Determination. The described traits were initially studied considering the following univariate repeatability animal model:

$$
y_{h k l i j}=H T D_{h}+D I M_{k}+a g e_{l}+p_{i}+a_{i}+e_{h k l i j},
$$

where the $j$ th observation $\left(y_{h k l i j}\right)$ of animal $i$ was explained by the effect of the $h$ th herd test day $(H T D)$ combination class, the $k$ th class of DIM, and the lth class of age, in addition to the permanent environmental $\left(p_{i}\right)$ and additive genetic $\left(a_{i}\right)$ effects of animal $i$. Herd test day included 145 levels, and DIM included 7 levels defined by the month of lactation (i.e., month after parturition). Finally, $e_{h k l i j}$ was a random residual term. The age of the ewe included 6 classes: the first class included records from ewes under 1 yr of age, the second from 1-yr-old females, the third from 2-yrold females, the fourth from 3- to 4-yr-old females, the fifth from females over $4 \mathrm{yr}$ of age, and the sixth from females whose age was not known (100 records). Priors for these systematic effects were uniform. Permanent environmental and additive genetic effects were fitted as factors for which these normal distributions were assumed as priors:

$$
\begin{gathered}
\mathbf{p} \mid \sigma_{p}^{2} \sim N\left(0, \sigma_{p}^{2} \times \mathbf{I}_{n}\right) \text { and } \\
\mathbf{a} \mid \sigma_{a}^{2} \sim N\left(0, \sigma_{a}^{2} \times \mathbf{A}\right)
\end{gathered}
$$

where $\sigma_{p}^{2}$ and $\sigma_{a}^{2}$ were the corresponding variances for the respective effects, $\mathbf{I}_{n}$ was the identity matrix of di- 
mension equal to the number of animals with records $(n)$, and $\mathbf{A}$ was the numerator relationship matrix between animals in the pedigree. Finally, the residuals (e) were assumed to follow this normal distribution:

$$
\mathbf{e} \mid \sigma_{e}^{2} \sim N\left(0, \sigma_{e}^{2} \times \mathbf{I}_{m}\right)
$$

where $\mathbf{I}_{m}$ is the identity matrix of dimension $m$ (i.e., number of records in the data set). Variance components were a priori assumed to follow a uniform distribution across all of the positive real numbers.

This univariate model was employed to test for additive genetic determination of each trait. For this purpose 2 criteria were used: the deviance information criterion (DIC; Spiegelhalter et al., 2002) was computed both for the model including the additive genetic effect and for the reduced model without this effect, and the Bayes factor (BF; Kass and Raftery, 1995) was computed between a model assuming the hypothesis of additive genetic standard deviation equal to zero and another considering this standard deviation greater than zero but smaller than the crude total phenotypic standard deviation presented in Table 1. For the calculation of $\mathrm{BF}$, a different parameterization of the above model was used:

$$
y_{h k l i j}=H T D_{h}+D I M_{k}+a g e_{l}+p_{i}+u_{i} \times \sigma_{a}+e_{h k l i j},[2]
$$

where $\mathbf{u} \mid \mathbf{A}, 1 \sim N(0,1 \times \mathbf{A})$ and $\sigma_{a}$ is the additive genetic standard deviation of the trait. This parameterization was needed because BF was computed using the definition by Garcia-Cortes et al. (2001), Varona et al. (2001), and Verdinelli and Wasserman (1995). GarciaCortes et al. (2001) showed, relying on the combination of continuous and discrete distributions through the usage of theory of generalized functions, that BF against the null hypothesis is equal to the ratio between the marginal prior and the marginal posterior densities of the parameter of interest, both evaluated at the parameter value under the null hypothesis (in our case, this hypothesis is that the additive genetic standard deviation is equal to zero):

$$
B F=\frac{p\left(\mathbf{y} \mid \sigma_{a} \neq 0\right)}{p\left(\mathbf{y} \mid \sigma_{a}=0\right)}=\frac{p\left(\sigma_{a}=0\right)}{p\left(\sigma_{a}=0 \mid \mathbf{y}\right)}
$$

Marginal posterior density evaluation can be accomplished within a Markov chain Monte Carlo (MCMC) approach by averaging conditional posterior density values at zero across all MCMC rounds (Garcia-Cortes et al., 2001). For the parameter value under the null hypothesis to be defined in this conditional density, the prior distribution must include it in its domain. We used this alternative parameterization because considering zero value in the domain of the prior for the additive genetic variance, under standard model [1], requires an equivalent model in which additive genetic structure is absorbed in the residual term. This makes inference an extremely demanding task in terms of needed computational resources, even for a low number of records. This equivalent model is needed to avoid sampling of BV, which cannot be accomplished under the considered prior assumptions because the normal distribution (usual prior for the BV) is not defined when variance is zero.

In our particular case, under the presented alternative parametrization, the prior distribution for $\sigma_{a}$ was a uniform between zero (included in its domain) and the total crude phenotypic standard deviation (i.e., empirical prior). Note that because priors for genetic variability are different between models [1] and [2], these models cannot be said to be equivalent. For the remaining parameters in model [2], the same set of prior assumptions as in model [1] was assumed.

This model parametrization and prior assumptions made the fully conditional posterior distribution of the additive genetic standard deviation proportional to a truncated normal distribution, with the mean equal to

$$
\frac{\sum_{j=1}^{m} e_{j}^{*} \times\left(\mathbf{z}_{j}^{\prime} \mathbf{u}\right)}{\sum_{j=1}^{m}\left(\mathbf{z}_{j}^{\prime} \mathbf{u}\right)^{2}}
$$

and variance

$$
\frac{\sigma_{e}^{2}}{\sum_{j=1}^{m}\left(\mathbf{z}_{j}^{\prime} \mathbf{u}\right)^{2}}
$$

the truncation points would be zero and the total crude phenotypic standard deviation of the studied trait. In the equations above, $e_{j}^{*}$ was the value obtained after correcting the $j$ th record for all the effects in the model except the complete additive genetic effect (i.e., $u_{i} \times$ $\left.\sigma_{a}\right)$, and $\mathbf{z}_{j}^{\prime}$ was a vector linking the $j$ th record to the appropriate additive genetic effect. This vector would be all zeros except for the position corresponding to the animal producing the $j$ th record, which would be 1 .

Differences in DIC of more than 7 units were considered as important (Spiegelhalter et al., 2002). With 
regard to BF, according to Jeffreys (1984), values lower than 1 support the null hypothesis; in this case, the trait does not show additive genetic variation and values greater than 3.16 indicate substantial evidence in favor of the alternative hypothesis (i.e., the trait has an additive genetic determination).

Multivariate Models for Estimating Correlations Between Traits. In a second step, genetic correlations between traits were estimated. For this purpose, 2 multivariate analyses were conducted, implementing model [1] in its multivariate version. In this case, the involved traits were assumed to jointly follow a multivariate normal distribution as well as the additive genetic, permanent environmental, and residual effects. For these effects, the corresponding prior distributions were

$$
\begin{gathered}
\mathbf{a} \mid \mathbf{G}_{0}, \mathbf{A} \sim M V N\left(0, \mathbf{G}_{0}, \otimes \mathbf{A}\right), \\
\mathbf{p} \mid \mathbf{P}_{0} \sim N\left(0, \mathbf{P}_{0}, \otimes \mathbf{I}_{n}\right), \text { and } \\
\mathbf{e} \mid \mathbf{R}_{0} \sim N\left(0, \mathbf{R}_{0}, \otimes \mathbf{I}_{m}\right),
\end{gathered}
$$

where $\mathbf{G}_{0}, \mathbf{P}_{0}$, and $\mathbf{R}_{0}$ were the corresponding variancecovariance matrices between the involved traits, and $\mathbf{a}$, $\mathbf{p}$, and $\mathbf{e}$ were, respectively, vectors of dimension equal to the number of animals in the pedigree $(n$ and $m)$ times the number of traits considered.

In the first multivariate analysis, individual FA and production traits were considered, and in the second analysis, groups and ratios of FA were jointly studied with production traits. In all of these analyses, information provided by the univariate analyses was considered (i.e., when DIC and BF did not support the hypothesis of a trait showing additive genetic variation, the additive genetic effect was not included in the model for this particular trait in the multivariate analyses) and all the individual variation was allocated to the permanent environmental effect.

MCMC Implementation. The parameters in the model were estimated using a Bayesian method implemented via an MCMC algorithm, in this case a Gibbs sampling. The Gibbs2f90 program (Misztal, 1999) was used to estimate parameters from model [1], and this program was properly modified for the alternative parameterization in model [2]. Details on theory involving a Bayesian MCMC analysis of the presented models can be found in Sorensen and Gianola (2002) for model [1] and in Varona et al. (1997) for model [2]. The MCMC chains consisted of 40,000 rounds for the univariate analysis and 1 million for the multitrait analysis, dis- carding the first $10 \%$ as a burning period. Afterward, to compute moments of marginal distributions, only $5 \%$ of the samples were used in the univariate analyses, retaining 1 sample every 20 iterations. In the multivariate analysis, $10 \%$ of the samples were used, retaining 1 sampled value every 100 iterations. Convergence of the chains was assessed both by visual inspection of their trace plots and by checking that the effective sample size was big enough.

\section{RESULTS AND DISCUSSION}

\section{Repeatabilities, Heritabilities, and Ratios of Permanent Variance to Phenotypic Variance}

$\boldsymbol{S F A}$. Both BF and DIC criteria were evidence of the existence of additive genetic variation for all the SFA (Table 2), but the magnitude of the estimated heritabilities for these traits was very low, the highest figure being 0.08 for both $\mathrm{C} 18: 0$ and C14:0. For this group of FA, the genetic component is the major factor determining individual variation of the trait, as can be seen by comparing heritabilities and ratios of permanent environmental variance to phenotypic variance. Despite the fact that all of the studied SFA showed significant additive genetic variance when they were studied individually, as a group they did not. This could be explained by the fact that this group included FA from different origin, and between them genetic correlations different from zero could exist, being in some cases of the opposite sign. Blending all together would reduce the overall genetic variance of the group. In fact, when the SFA group was defined considering only those FA individually studied, a value of heritability statistically not different from zero was still estimated.

Low heritability estimates for this group of FA as well as their low coefficient of variation would prevent selection programs from being considered as an alternative to modifying the content of SFA in Churra milk. Previous studies in dairy cattle have shown much higher heritabilities for those SFA that we studied. Estimates for the Dutch Holstein population (Stoop et al., 2008) are particularly high, with values for intraherd heritability as high as 0.71 for C10:0. Soyeurt et al. (2007) estimated heritability values for a blend of Belgium breeds ranging from 0.09 for C12:0 to 0.28 for C18:0. Recently, for the US Holstein population, Bobe et al. (2008) estimated heritabilities for the proportion of different SFA, in comparison with the total amount of FA in milk, were similar to those reported for the Belgian cattle population. However, for the Italian HolsteinFriesian population, much lower heritabilities have been estimated for SFA (0.07, 0.03, and 0.08 for C14:0, C16:0, and C18:0, respectively; Mele et al., 2009). 
Table 2. Univariate estimates of heritability $\left(\mathrm{h}^{2}\right)$, ratio of permanent environmental variance to phenotypic variance $\left(\mathrm{p}^{2}\right)$, repeatability $\left(\mathrm{r}^{2}\right)$, and results of the model choice tests: difference in deviance information criteria (DIC diff.) between genetic and nongenetic models and Bayes factor $(\mathrm{BF})$ between nongenetic and genetic models

\begin{tabular}{|c|c|c|c|c|c|}
\hline Trait $^{1}$ & \multicolumn{2}{|c|}{ Genetic model $^{2}$} & $\frac{\text { Nongenetic model }}{r^{2}}$ & \multicolumn{2}{|c|}{ Model choice criteria } \\
\hline $\mathrm{C} 8: 0$ & $0.05(0.02)$ & $0.03(0.02)$ & $0.07(0.01)$ & -22.18 & $5.50 \mathrm{E}+09$ \\
\hline C12:0 & $0.07(0.03)$ & $0.06(0.03)$ & $0.13(0.02)$ & -22.47 & $5.10 \mathrm{E}+13$ \\
\hline C14:0 & $0.08(0.03)$ & $0.04(0.02)$ & $0.11(0.01)$ & -30.35 & $4.64 \mathrm{E}+11$ \\
\hline C16:0 & $0.04(0.01)$ & $0.01(0.01)$ & $0.04(0.01)$ & -25.53 & $2.33 \mathrm{E}+05$ \\
\hline $\mathrm{C} 18: 1$ trans -11 & $0.02(0.01)$ & $0.02(0.01)$ & $0.04(0.01)$ & -11.43 & $3.39 \mathrm{E}+00$ \\
\hline C18:1 cis-9 & & & $0.05(0.01)$ & -2.00 & $5.32 \mathrm{E}-02$ \\
\hline $\mathrm{C} 18: 2$ cis- 9 , cis- 12 & $0.11(0.04)$ & $0.07(0.03)$ & $0.17(0.02)$ & -24.79 & $5.47 \mathrm{E}+35$ \\
\hline C18:3 cis-9, cis-12, cis-15 & & & $0.06(0.01)$ & -3.54 & 3.99E-01 \\
\hline C18:2 cis-9, trans-11 (CLA) & & & $0.04(0.01)$ & -0.74 & $1.03 \mathrm{E}-01$ \\
\hline SFA & & & $0.08(0.01)$ & -1.22 & $1.50 \mathrm{E}-02$ \\
\hline MUFA & & & $0.06(0.01)$ & -1.94 & $4.57 \mathrm{E}-02$ \\
\hline Milk yield $(\mathrm{mL} / \mathrm{d})$ & $0.16(0.06)$ & $0.31(0.06)$ & $0.46(0.02)$ & -11.36 & $2.29 \mathrm{E}+07$ \\
\hline
\end{tabular}

${ }^{1} \mathrm{CLA}=$ conjugated linoleic acid; SFA = saturated fatty acids (FA); MUFA = monounsaturated FA; PUFA = polyunsaturated FA. The content of FA is expressed as a percentage in relation to the total amount of FA. n-6 includes C18:2 cis-9, cis-12, C18:3 cis-6, cis-9, cis-12, and C20:4 cis-5, cis-8, cis-11, cis-14; n-3 includes C18:3 cis-9, cis-12, cis-15.

${ }^{2}$ Blanks correspond to traits for which a nongenetic model is statistically preferable, so no heritability estimates are reported. For those traits showing significant additive genetic variation, the reported parameter estimates under the genetic model correspond to those obtained under model 2 (see equation [2]); better mixing was observed when this genetic model was used.

Unsaturated Fatty Acids. With regard to MUFA, only the content of C18:1 trans-11 (trans-vaccenic acid) could be said to have an additive genetic determination, and again the estimated heritability value was very low (Table 2). When all the MUFA were studied as a group, no significant additive genetic variation was observed. This result made sense because approximately $89 \%$ of this group was composed of the individual FA shown in Table 2, and among them only C18:1 trans-11, which represented a mere $11 \%$ of the group, had a significant additive genetic determination; however, its heritability was very low.

Among PUFA, the hypothesis of traits showing additive genetic variation could be supported only for C18:2 cis-9,cis-12 (cis isomer of linoleic acid). The estimated heritability for this FA was 0.11 (0.04) (Table 2). Also, it was observed that the magnitude of this heritability was greater than the respective value of the ratio of the permanent environmental variance to the total variance. For the PUFA group, a significant additive genetic determination was observed despite the fact that only 1 of the FA included in this group (C18:2 cis-9,cis-12) showed a significant additive genetic variance. It should be noted that this FA represented approximately $53 \%$ of the group.
In general, except for $\mathrm{C} 18: 1$ trans-11 and C18:2 cis9 , cis-12, the content of unsaturated FA (UFA) cannot be said to be heritable in the Churra breed. In dairy cattle, lower heritabilities for UFA than for SFA have been estimated but, contrary to our results, the magnitude of these estimates is still significantly different from zero. Stoop et al. (2008) reported values around 0.25 for different MUFA and polyunsaturated 18-carbon-chain FA, and Soyeurt et al. (2007) estimated heritability values of 0.15 for C18:1 and CLA. In our case, observing a significant value of heritability for trans-vaccenic (C18:1 trans-11) and cis-linoleic (C18:2 cis-9,cis-12) acids could be said to be unexpected, particularly after considering that these FA are generated or modified by rumen bacteria and that they cannot be obtained by endogenous biosynthesis. This result could support the hypothesis presented in the introduction about genetic variability in the determination of rumen microbial population structure and activity. To our knowledge, no studies have investigated this hypothesis, and indeed more research is needed to support it; however, evidence of individual variability in the composition of ruminant bacteria has been previously reported (Swain et al., 1996) by considering variations in the bacteriophage population. 


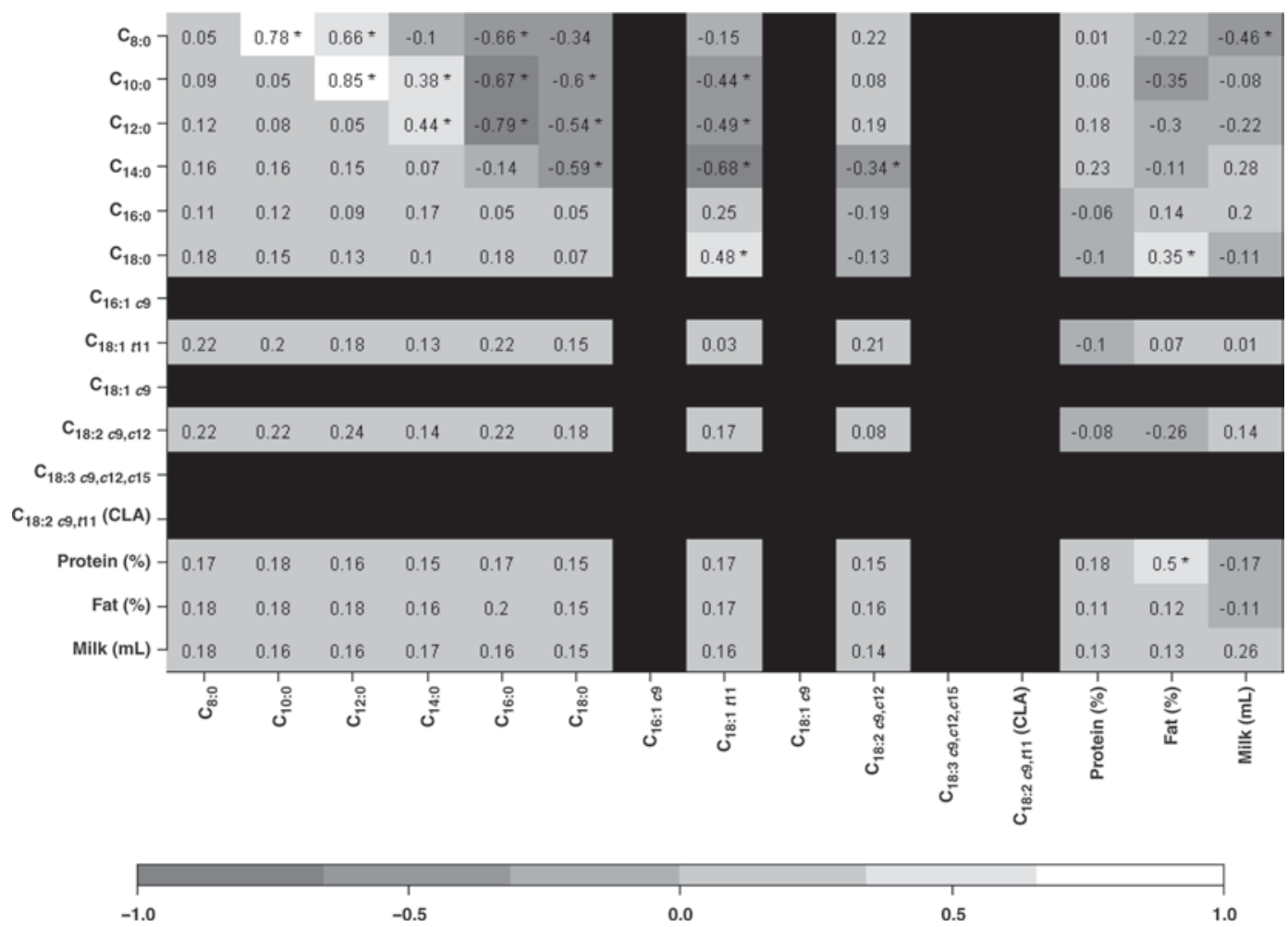

Figure 1. Genetic correlations between individual fatty acids and production traits. Upper triangle $=$ marginal posterior means; lower triangle $=$ marginal posterior standard deviations; diagonal = multitrait heritabilities. Asterisks represent correlation estimates for which the $95 \%$ highest posterior density region does not include zero. Black rows and columns correspond to those traits not showing significant additive genetic variance in the univariate analysis. $c=$ cis; $t=$ trans.

For the particular cases of CLA and the ratio CLA:C18:1 trans-11, no significant additive genetic variation was observed. This result is in agreement with the low number of allelic variants so far observed in the gene coding this enzyme across several sheep breeds, including Churra: only 4 SNP have been observed and they were located in noncoding regions (GarcíaFernández et al., 2009).

Production Traits. The genetic model was always supported by the data and heritability estimates were in line with estimated values previously reported for the respective traits in this breed (Othmane et al., 2002; El-Saied et al., 2006). However, the magnitude of the estimated heritabilities for these traits was lower than those reported for other dairy sheep breeds performing under less variable environments; for example, Lacaune (Barillet and Boichard, 1987). In dairy cattle, heritability estimates for FA content are in general lower than those for production traits. In agreement with this observation, we obtained in Churra lower heritability estimates for FA than for production traits.

To our knowledge, only one study has investigated the genetic determination of FA content in sheep milk (Carta et al., 2008) in a crossbred population between
Lacaune and Sardinia breeds. Within this project, QTL regions involved in the determination of different FA contents and ratios were detected. But no reports on the genetic determination of traits according to the genetic infinitesimal model have been published.

\section{Genetic Correlations}

Individual FA and Production Traits. In general, the studied SFA of medium chain length (C8:0-C14:0) were moderately genetically positively correlated to each other (Figure 1). The genetic correlation between these SFA and the studied long-chain SFA (C16:0, C18:0) were in general lower in magnitude than the previous correlations, but negative. The genetic correlation between C16:0 and C18:0 could not be said to be different from zero. Similar results were obtained for Dutch (Stoop et al., 2008) and Belgian (Soyeurt et al., 2007) cattle populations.

Regarding the genetic correlation between the considered UFA (C18:2 cis-9,cis-12 and C18:1 trans-11-those presenting significant additive genetic variation in the univariate analyses), given its 95\% highest posterior density interval, it could not be said to be different from 
zero. Previous studies in dairy cattle have, in general, reported positive estimates for genetic correlations between UFA (Soyeurt et al., 2007; Stoop et al., 2008). In our case, the only genetic correlations between SFA and UFA with relevant magnitudes were observed between C10:0, C12:0, C14:0, C18:0, and C18:1 trans-11 and between C14:0 and C18:2 cis-9,cis-12 (Figure 1). There does not seem to be congruency among previous studies in dairy cattle for these correlations. Mele et al. (2009) reported a genetic correlation of -0.91 between C18:0 and C18:1 trans-11, but Stoop et al. (2008) reported a value of 0.05 .

The estimates of the genetic correlation between individual FA and production traits could not be said to be different from zero, except between fat percentage and C18:0 and milk yield and C8:0 (Figure 1). In dairy cattle, genetic correlations between fat and protein percentages and FA are higher in magnitude than our estimates. It seems that SFA are positively correlated to fat percentage (except C14:0), and protein percentage and UFA are negatively correlated to both fat and protein percentage (Soyeurt et al., 2007).

Finally, regarding correlations between production traits, negative values were observed between milk yield and both percentage of protein and fat, but their $95 \%$ highest posterior density included zero. Genetic correlation showed a moderate positive value of 0.50 between these 2 percentages.

When comparing the estimated heritabilities from univariate and multivariate analyses, fairly high agreement was observed for all FA and percentages of fat and protein in milk; however, this was not the case for milk yield. A much higher value of heritability was observed for this trait from the multitrait (MT) analysis. As could be expected, for all the traits the marginal posterior standard deviations for heritabilities from the MT analysis were smaller than those from univariate analyses (result not shown).

Groups of FA and Production Traits. The only group of FA showing additive genetic variation in the univariate analyses was PUFA; however, in the multivariate analysis its estimated heritability was zero and the magnitude of the correlations between this FA and production traits were close to zero as well. Thus, any further discussion of these genetic correlations is unnecessary. The genetic correlations between production traits estimated in this analysis were similar to those obtained from the MT analysis involving individual FA and production traits, which were 0.6(0.13), $-0.12(0.16)$, and $-0.07(0.17)$ between fat and protein percentages, between fat percentage and milk yield $(\mathrm{mL})$, and between protein percentage and milk yield $(\mathrm{mL})$, respectively.
In this second multivariate analysis, large discrepancies were also observed between the estimated heritability for milk yield and that obtained in the univariate analysis. Therefore, to assess the influence of FA and their groups and ratios on the heritability estimate for milk yield, a third analysis involving only the 3 production traits was conducted. In this analysis, the heritability estimate for milk yield was slightly higher than that observed in the univariate analyses (result not shown) but lower than that in Figure 1 and that obtained in the second MT analysis. Thus, the discrepancies may be a consequence of the effect of the correlations between FA, FA ratios and groups, and milk yield.

Despite this discussion about genetic correlations between FA contents, groups, and ratios of FA and production traits, the difficulty in estimating these parameters must be addressed. This is reflected in the high magnitude of their posterior standard deviations and in the observed discrepancies between estimates for the same parameter across studies.

\section{General Discussion}

In most previous studies in dairy cattle, FA ratios involving a cis-9 UFA and its respective saturated counterpart, which are regarded as proxies of $\Delta^{9}$-desaturase activity, have been shown to present additive genetic variation. This results in a general increase in additive genetic variance for endogenous biosynthesized FA, as well as in additive genetic covariance between them. We did not observe this genetic variation, which could well explain our low estimates of heritabilities, in particular for UFA. Another explanation for the general low magnitude of the estimated heritabilities, repeatabilities, and correlations in our population could be related to the fact that in Churra commercial milk production systems, even during milking periods, it is common to alternate indoor feeding with outdoor pasture usage. This practice generates additional variation because of interactions between individual animals and feeding systems (i.e., $\mathrm{G} \times \mathrm{E}$ interaction), which cannot be taken into account by our model. This additional source of variation would be important in traits highly affected by diet. So, at best, our estimates could be interpreted as an average of the parameters in all the range of possible environments. But, in the worst case, the extra nonconsidered variation, because of these putative interactions, could be assigned to the residual terms, reducing the magnitude of the estimated heritabilities and repeatabilities. The presence of these $\mathrm{G}$ $\times \mathrm{E}$ interactions is particularly likely in our sample given that a wide range of nongenetic factors (e.g., several lactations, long lactation periods, and record- 
ing throughout the year) are covered. An alternative to reduce this putative interaction would be to reduce the range of nongenetic factors, as has been done by Stoop et al. (2008), but then the sample would not fully reflect the complete environmental variation in that population.

In this study, our interest was in estimating genetic parameters for FA composition (i.e., FA in fat) to assess the possibility of modifying FA profile by selective breeding. An alternative selection criterion could be the amount of FA in milk. For this trait, it would be expected to have a higher heritability because genetic variation of fat percentage would be involved. We observed this fact when the studied traits were FA compositions (our current traits) multiplied by fat percentage of each particular sample. This criterion would be more appropriate for selection to increase the total amount of a particular FA, but not for altering FA profile, because in using these traits higher correlations would exist between the selection criteria; fat percentage is a common factor to all of the traits. Then it would not be possible to modify the amount of different FA in opposite directions.

\section{CONCLUSIONS}

In general, very low additive genetic variance was estimated for the percentages of the different FA in Churra sheep fat milk. This result does not allow a selection breeding program based on classical animal evaluations to be considered for modifying the FA profile in this breed's milk under current production conditions.

\section{ACKNOWLEDGMENTS}

This research was supported by the Spanish Ministry of Science Project AGL2005-04321 and by a grant (GR.43/2008) for excellence research groups of the Castile and León regional government. The authors appreciate the contributions of Catherine Martin (Léon, Spain) in the English revision of the manuscript and of two anonymous referees.

\section{REFERENCES}

Barillet, F., and D. Boichard. 1987. Studies on dairy production of milking ewes. 1. Estimates of genetic parameters for total milk composition and yield. Genet. Sel. Evol. 19:459-474.

Bligh, E. G., and W. J. Dyer. 1959. A rapid method of total lipid extraction and purification. Can. J. Biochem. Physiol. 37:911917.

Bobe, G., J. A. M. Bormann, G. L. Lindberg, A. E. Freeman, and D. C. Beitz. 2008. Short communication: Estimates of genetic variation of milk fatty acids in US Holstein cows. J. Dairy Sci. 91:1209-1213.
Cabiddu, A., M. Decandia, M. Addis, G. Piredda, A. Pirisi, and G. Molle. 2005. Managing Mediterranean pastures in order to enhance the level of beneficial fatty acids in sheep milk. Small Rumin. Res. 59:169-180

Carta, A., S. Casu, M. G. Usai, M. Addis, M. Fiori, A. Fraghi, S. Miari, L. Mura, G. Piredda, L. Schibler, T. Sechi, J. M. Elsen, and F. Barillet. 2008. Investigating the genetic component of fatty acid content in sheep milk. Small Rumin. Res. 79:22-28.

De La Fuente, L. F., E. Barbosa, J. A. Carriedo, C. Gonzalo, R Arenas, J. M. Fresno, and F. San Primitivo. 2009. Factors influencing variation of fatty acid content in ovine milk. J. Dairy Sci. 92:3791-3799.

De La Fuente, L. F., J. A. Baro, and F. San Primitivo. 1995. Breeding progamme for the Spanish Churra sheep breed. Pages 165-172 in Strategies for Sheep and Goat Breeding. Vol. 11. D. Gabina, ed. Centre International de Hautes Etudes Agronomiques Mediterraneennes, Zaragoza, Spain.

El-Saied, U. M., L. F. de la Fuente, and F. San Primitivo. 2006. Lifetime traits comparison between annual and accelerated lambing systems for dairy ewes. Livest. Sci. 101:180-190.

Garcia-Cortes, L. A., C. Cabrillo, C. Moreno, and L. Varona. 2001. Hypothesis testing for the genetic background of quantitative traits. Genet. Sel. Evol. 33:3-16.

García-Fernández, M., B. Gutiérrez-Gil, E. García-Gámez, and J.-J. Arranz. 2009. Genetic variability of the stearoyl-CoA desaturase gene in sheep. Mol. Cell. Probes 23:107-111.

Gómez-Cortés, P., P. Frutos, A. R. Mantecón, M. Juarez, M. A. de la Fuente, and G. Hervás. 2008. Addition of olive oil to dairy ewe diets: Effect on milk fatty acid profile and animal performance. J. Dairy Sci. 91:3119-3127.

Jeffreys, H. 1984. Theory of Probability. 3rd ed. Clarendon Press, Oxford, UK.

Kass, R. E., and A. E. Raftery. 1995. Bayes factors. J. Am. Stat. Assoc. 90:773-795.

Khanal, R. C., and T. R. Dhiman. 2004. Biosynthesis of conjugated linoleic acid (CLA): A review. Pak. J. Nut. 3:72-81.

Mele, M., G. Conte, B. Castiglioni, S. Chessa, N. P. P. Macciotta, A. Serra, A. Buccioni, G. Pagnacco, and P. Secchiari. 2007. Stearoylcoenzyme A desaturase gene polymorphism and milk fatty acid composition in Italian Holsteins. J. Dairy Sci. 90:4458-4465.

Mele, M., R. Dal Zotto, M. Cassandro, G. Conte, A. Serra, A. Buccioni, G. Bittante, and P. Secchiari. 2009. Genetic parameters for conjugated linoleic acid, selected milk fatty acids, and milk fatty acid unsaturation of Italian Holstein-Friesian cows. J. Dairy Sci. 92:392-400.

Milanesi, E., L. Nicoloso, and P. Crepaldi. 2008. Stearoyl CoA desaturase (SCD) gene polymorphisms in Italian cattle breeds. J. Anim. Breed. Genet. 125:63-67.

Misztal, I. 1999. Complex models, more data: Simpler programming? Pages 32-41 in Computational Cattle Breeding Workshop. Interbull, Tuusula, Finland.

Mitchell, P. L., and R. S. McLeod. 2008. Conjugated linoleic acid and atherosclerosis: Studies in animal models. Biochem. Cell Biol. 86:293-301.

Othmane, M. H., L. F. De La Fuente, J. A. Carriedo, and F. San Primitivo. 2002. Heritability and genetic correlations of test day milk yield and composition, individual laboratory cheese yield, and somatic cell count for dairy ewes. J. Dairy Sci. 85:2692-2698.

Pascal, G. 1996. Recommended daily intake of lipids and fatty acids. OCL-Ol. Corps. Gras. Li. 3:205-210.

Sorensen, D., and D. Gianola. 2002. Likelihood, Bayesian, and MCMC Methods in Quantitative Genetics. Springer, New York, NY.

Soyeurt, H., A. Gillon, S. Vanderick, P. Mayeres, C. Bertozzi, and N. Gengler. 2007. Estimation of heritability and genetic correlations for the major fatty acids in bovine milk. J. Dairy Sci. 90:44354442.

Spiegelhalter, D. J., N. G. Best, B. R. Carlin, and A. van der Linde. 2002. Bayesian measures of model complexity and fit. J. Roy. Stat. Soc. B Met. 64:583-616.

Stoop, W. M., J. A. M. van Arendonk, J. M. L. Heck, H. J. F. van Valenberg, and H. Bovenhuis. 2008. Genetic parameters for major 
milk fatty acids and milk production traits of Dutch HolsteinFriesians. J. Dairy Sci. 91:385-394.

Swain, R. A., J. V. Nolan, and A. V. Klieve. 1996. Natural variability and diurnal fluctuations within the bacteriophage population of the rumen. Appl. Environ. Microbiol. 62:994-997.

Taniguchi, M., H. Mannen, K. Oyama, Y. Shimakura, A. Oka, H. Watanabe, T. Kojima, A. Komatsu, G. S. Harper, and S. Tsuji. 2004. Differences in stearoyl-CoA desaturase mRNA levels between Japanese Black and Holstein cattle. Livest. Prod. Sci. 87:215220 .

Tricon, S., G. C. Burdge, S. Kew, T. Banerjee, J. J. Russell, R. F. Grimble, C. M. Williams, P. C. Calder, and P. Yaqoob. 2004. Effects of cis-9,trans-11 and trans-10,cis-12 conjugated linoleic acid on immune cell function in healthy humans. Am. J. Clin. Nutr. 80:1626-1633.
Tricon, S., and P. Yaqoob. 2006. Conjugated linoleic acid and human health: A critical evaluation of the evidence. Curr. Opin. Clin. Nutr. Metab. Care 9:105-110.

Varona, L., L. A. Garcia-Cortes, and M. Perez-Encisco. 2001. Bayes factors for detection of quantitative trait loci. Genet. Sel. Evol. 33:133-152.

Varona, L., C. Moreno, L. A. Garcia-Cortes, and J. Altarriba. 1997. Model determination in a case of heterogeneity of variance using sampling techniques. J. Anim. Breed. Genet. 114:1-12.

Verdinelli, I., and L. Wasserman. 1995. Computing Bayes factors using a generalization of the Savage-Dickey density ratio. J. Am. Stat. Assoc. 90:614-618. 